Data Standards Assurance Program (2010) has mandated that electronic Discharge Summary (DS) should be sent out from medical teams to the appropriate GP within $24 \mathrm{~h}$ of the patient being discharged and the patient should be sent home with one copy of DS in their hands. In 2013, 60\% of the Renal Ward discharge summaries were being sent out in time.

Assessment of problem and analysis of its causes We interviewed doctors, nurses and pharmacists to get a better understanding of the process that led to DS being posted to GPs. We identified following factors that led to delays: poor communication within the team and lack of ownership of this task. We held a stakeholder meeting for brain storming and shared ideas to improve the existing process. We developed junior doctor rota describing who is responsible for the DS completion and agreed that the patient's nurse was responsible for ensuring that patient did not leave without DS.

Intervention The new process for writing DC comprised of a number of steps. On call junior doctor for the week is in charge of getting information from the senior nurse on who will need the DS on a daily basis; the same person is in charge of completing DS, printing it and giving it to the patient's nurse who was not allowed to discharge patent without a DS. We allocated new place for the storage of GP copies of DS to trigger action from administrative staff to post DS to GPs. We ensured that all team members were aware of the new system by attending handover sessions.

Study design Observational study.

Strategy for change We attended 'away days'for all levels of nurses and junior doctors induction and informed them of the new system. We encouraged all team members to approach project leads if they identify new problems as those were to be our new PDSA cycles. We aimed to have $>90 \%$ DC summaries done timely over a 16 week period. The National standard is $95 \%$.

Measurement of improvement We kept a record of every DS completed. For those not completed a mini root cause analysis was done to investigate the cause of the delay. Data analysed weekly and results shared with all staff.

Effects of changes The effects of changes were better quality of patient care as timely DS done (one of six domains of quality) and improved communication between professionals in tertiary care and GPs. We achieved and sustained $>90 \%$ target (Figure 1).
Lessons learnt This project taught me the importance of a multidisciplinary approach when planning change. We need a desire and commitment to change and on planning the improvements. It is crucial to see problem through different professionals views as this aids problem analysis. Next time, I would involve a patient or a parent to oversee our work.

Message for others Understanding problem and the process before implementing change is crucial for success. Making sure that all team members are aware of project and evaluating feedback is important for sustainability. This improvement is important for quality of care we provide as it addresses one of six quality care domains. Discuss each failure with the team and perform new PDSA for each problem identified.

\section{G597 AUDIT AND RE-AUDIT OF DISTRACTIONS DURING PRESCRIBING IN A PAEDIATRIC CRITICAL CARE UNIT}

${ }^{1} \mathrm{~K}$ Chan, ${ }^{2} \mathrm{~K}$ Sadasivam, ${ }^{2} \mathrm{~N}$ Edmonds. ${ }^{1}$ Barts and the London School of Medicine and Dentistry, Queen Mary University of London, London, UK; ${ }^{2}$ Barts Health NHS Trust, The Royal London Hospital, London, UK

\subsection{6/archdischild-2015-308599.546}

Context A 6-beded Paediatric Critical Care Unit (PCCU) at Royal London Hospital (UK) with the involvement of consultants, registrars and pharmacists at PCCU.

Problem A previous audit at PCCU showed that prescribing errors occurred at a frequency of 0.02 per PCCU bed days in 2013, with 3 cases of serious drug dose error, incorrect adjustment for renal impairment and electrolyte replacement error. A dedicated prescribing area was introduced in 2013 but the effectiveness was undetermined. Upon observation of prescribing practice at PCCU, it was noticed that prescribers were distracted frequently, which might be contributory to the increase in prescribing errors.

Assessment of problem and analysis of its causes An audit was designed to observe prescribers and to quantify the distractions. The effectiveness of the prescribing area was measured in terms of its usage frequency, prescribing pattern of PCCU doctors and the nature of distractions during prescribing. PCCU doctors were involved, and it was noted that there was high frequency of distractions during prescribing. Interventions on areas such as

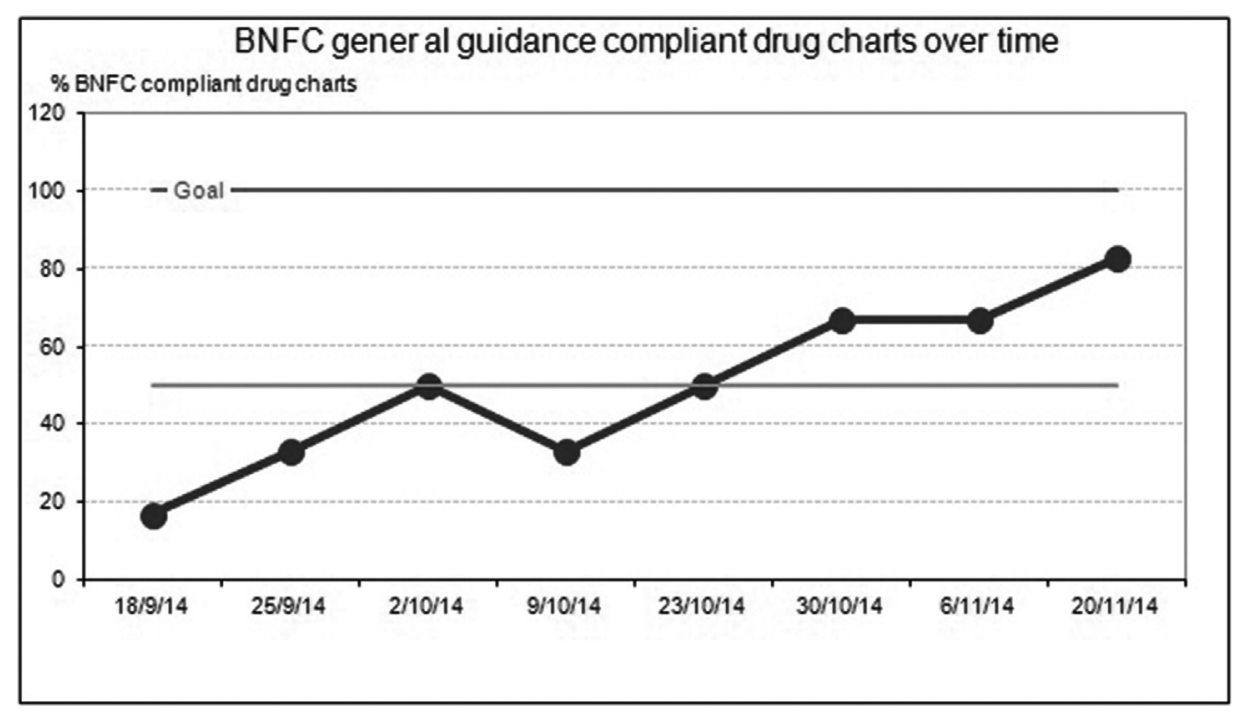


information provision, education and training, zero tolerance policy and a re-audit were introduced.

Intervention i. Information Provision

Prescribing tools were provided at the prescribing area:

- latest British National Formulary

- laminated common drug and infusion dosage charts

- spare drug and infusion charts

- stationery: a calculator and pens

ii. Education and Training

- Immediate feedback was given to staff

- Presentations were given at various staff meetings to raise awareness

- Safe prescribing practice was reinforced in induction programmes

iii. Zero Tolerance Policy

- Prominent signage was displayed signifying that the prescribing area was an undisturbed area

iv. Re-audit

A re-audit was carried out to assess the effectiveness of the interventions

Study design A 1-week prospective, non-blinded, observational audit was performed. The number and nature of distractions was recorded. Primary outcome was the frequency and nature of prescribing errors, which were identified by pharmacists. Interventions were implemented.

A 1-week re-audit was performed to assess the effectiveness of the interventions.

Strategy for change There was communication with the staff to devise feasible interventions, which were strongly supported by consultants and pharmacists at PCCU. The audit results were presented 3 times in different staff meetings to raise awareness and educate staff.

Measurement of improvement A 1-week re-audit was performed to assess the number of distractions and the frequency of prescribing errors.

Effects of changes Pre-intervention audit: There were 12 observable distractions and 8 prescribing mistakes before the interventions in one week. Distractions included ward duties and interruptions from colleagues, nurses and visitors.

Post-intervention re-audit: There were 0 observable distraction and 3 prescribing mistakes in the re-audit. The interventions also changed the culture of prescribing practice.

Lessons learnt Constant reinforcement of zero tolerance to prescribing errors and ongoing education were the key for the substantial change in prescribing practice. Modifications at the prescribing area effectively reduced observable distractions and prescribing errors.

The audit period was very short since this was the first audit on distractions and acted as a pilot study for future audits. Standardised and ongoing reviews with systematic parameters should be devised to ensure the adherence of prescribing practice and to monitor the frequency of prescribing errors.

Message for others Strong leadership is critical in implementing change and sustainability of system change is important. Education of safe prescribing practice is crucial and the most effective way is to bear patient's safety in mind, be mindful and always check the prescriptions before administering. 